\title{
ISOTOPY EQUIVALENCE CLASSES OF NORMAL ARCS IN $F \times I$
}

\author{
C. D. FEUSTEL
}

\begin{abstract}
Let $F$ be a compact 2-manifold and $I$ the closed unit interval. Let $\alpha$ and $\beta$ be arcs embedded in $F \times I$ such that $\alpha$ and $\beta$ meet the boundary of $F \times I$ in the boundary of $\alpha$ and $\beta$ respectively. Then we give necessary and sufficient conditions for the existence of an ambient isotopy, constant on the boundary of $F \times I$, moving $\alpha$ to $\beta$. We also obtain ambient isotopies of families of arcs properly embedded in $F \times I$.
\end{abstract}

I. Introduction. In [4] Martin shows that two arcs having common endpoints embedded in the interior of a 3-manifold $M$ are isotopic if and only if they are homotopic. In this paper we shall concern ourselves with the existence of ambient isotopies moving one properly embedded arc in $M$ to another. We shall restrict ourselves to the case when our 3-manifold $M$ is the product of a compact surface $F$ with the unit interval $I$.

All spaces in this paper are simplicial complexes and all maps are piecewise linear.

Before proceeding it will be useful to establish some notation. Let $X$ be a triangulated $n$-manifold. Then $\operatorname{bd}(X)$ is the $(n-1)$-submanifold of $X$ consisting of all $(n-1)$-simplexes which are faces of exactly one $n$-simplex. Let $Y$ be a manifold. An embedding $X$ in $Y$ is proper if $X \cap \operatorname{bd}(Y)=\operatorname{bd}(X)$. Throughout this paper $F$ will be a compact connected surface and $I$ will be the unit interval. We let $p: F \times I \rightarrow F$ be the natural projection map.

Let $\left\{\alpha_{i}: i=1, \cdots, n\right\}$ be a collection of disjoint arcs properly embedded in $F \times I$ such that $\operatorname{bd}\left(\alpha_{i}\right) \subset(F-\operatorname{bd}(F)) \times\{0,1\}$ and $p \operatorname{bd}\left(\alpha_{i}\right)=\left\{x_{i}\right\}$ for $i=1, \cdots, n$. Then we shall say that $\left\{\alpha_{i}: i=1, \cdots, n\right\}$ is a normal collection of arcs. An isotopy of a homeomorphism $h: X \rightarrow Y$ is a map $H: X \times I \rightarrow Y$ such that for $h_{t}=H \mid X \times\{t\}$ we have $h_{0}=h$ and $h_{t}$ is a homeomorphism onto $Y$. An isotopy of subspaces $Z_{1}$ and $Z_{2}$ in $X$ is an isotopy of the identity map on $X$ such that $h_{1}\left(Z_{1}\right)=Z_{2}$.

Let $\left\{\alpha_{i}: i=1, \cdots, n\right\}$ and $\left\{\beta_{i}: i=1, \cdots, n\right\}$ be normal collections of arcs in $F \times I$. Then $\left\{\alpha_{i}: i=1, \cdots, n\right\}$ is homeomorphically unknotted if there is a

Received by the editors August 15, 1972.

AMS (MOS) subject classifications (1970). Primary 55A25; Secondary 57A10.

(c) American Mathematical Society 1973 
homeomorphism $h: F \times I \rightarrow F \times I$ such that

(1) $h\left(\alpha_{i}\right)=\left\{x_{i}\right\} \times I$ for $i=1, \cdots, n$,

(2) $h \mid F \times\{0\} \cup \operatorname{bd}(F) \times I$ is the identity.

The collection $\left\{\alpha_{i}: i=1, \cdots, n\right\}$ is isotopically unknotted if the $\bigcup_{i=1}^{n} \alpha_{i}$ and $\bigcup_{i=1}^{n}\left\{x_{i}\right\} \times I$ are isotopic under a deformation constant on $\operatorname{bd}(F \times I)$. We shall say that the collections $\left\{\alpha_{i}: i=1, \cdots, n\right\}$ and $\left\{\beta_{i}: i=1, \cdots, n\right\}$ are homeomorphically equivalent if there is a homeomorphism $h: F \times I \rightarrow$ $F \times I$ such that

(1) $h\left(\alpha_{i}\right)=\beta_{i}$ for $i=1, \cdots, n$,

(2) $h \mid F \times\{0\} \cup \operatorname{bd}(F) \times I$ is the identity.

We shall say that the collections $\left\{\alpha_{i}: i=1, \cdots, n\right\}$ and $\left\{\beta_{i}: i=1, \cdots, n\right\}$ are isotopically equivalent if there is an isotopy of the subspaces $\bigcup_{i=1}^{n} \alpha_{i}$ and $\bigcup_{i=1}^{n} \beta_{i}$ which is constant on $\mathrm{bd}(F \times I)$.

A 3-manifold $M$ is irreducible if every 2-sphere embedded in $M$ bounds a 3-ball embedded in $M$. It is well known that $F \times I$ is irreducible if $F$ is not the 2-sphere.

We shall try to follow Waldhausen's principal of "induction on niceness". (See [6, p. 58].) That is, after we convince ourselves there is no obstruction to achieving some niceness, we take up our problem again assuming that niceness.

II. Useful results. In [3] we prove the following four theorems.

THEOREM 2.1. Let $h: F \times I \rightarrow F \times I$ be a homeomorphism such that $h \mid \operatorname{bd}(F) \times I \cup F \times\{0\}$ is the identity. Then there is an isotopy, constant on $\mathrm{bd}(F) \times I \cup F \times\{0\}$ of $h$ to the identity.

Let $\alpha$ be a normal arc embedded in $F \times I$. Then $\alpha$ is monotonic in $F \times I$ if $\alpha \cap F \times\{t\}$ is a single point for $t$ in $I$.

THEOREM 2.2. We assume that $F$ is not the 2-sphere. Let $\alpha$ be a normal arc in $M=F \times I$ such that $p \operatorname{bd}(\alpha)=\{x\}$. Then there is an isotopy of $M$ constant on $\operatorname{bd}(M)$ carrying $\alpha$ to a monotonic arc if and only if $\alpha$ is homeomorphically unknotted.

THEOREM 2.3. Let $\alpha$ be a normal arc in $M=F \times I$ such that $p \operatorname{bd}(\alpha)=\{x\}$. Then $\alpha$ is isotopically unknotted if and only if $\alpha$ is homeomorphically unknotted and the loop $p(\alpha)$ is homotopic to a point.

THEOREM 2.4. Let $F$ be a surface other than the 2-sphere. Let $F=$ $F_{1} \cup F_{2}$ and $F_{1} \cap F_{2}=c$ be a component of $\mathrm{bd}\left(F_{1}\right)$. Let $\alpha$ be a normal arc in $F_{1} \times I$. Then $\alpha$ is a homeomorphically unknotted in $F_{1} \times I$ if and only if it is homeomorphically unknotted in $F \times I$.

The following theorem is an immediate consequence of Theorem 5.2 in [1] and Lemma 3.4 in [3]. 
THEOREM 2.5. Let $F$ be a surface other than the projective plane, Klein bottle, torus, or 2-sphere. Let $A$ and $B$ be annuli properly embedded in $M=F \times I$ such that $\mathrm{bd}(A)=\mathrm{bd}(B)$ and $\mathrm{bd}(A) \cap F \times\{j\}$ is a non-nullhomotopic loop embedded in the interior of $F \times\{j\}$ for $j=0,1$. Then there is an isotopy constant on bd $(F \times I)$, carrying $A$ to $B$.

\section{Equivalence of knotted arcs.}

THEOREM 3.1. Let $\left\{\alpha_{i}: i=1, \cdots, n\right\}$ and $\left\{\beta_{i}: i=1, \cdots, n\right\}$ be normal collections of arcs in $M=F \times I$ which are homeomorphically equivalent. Suppose for some $t_{0}$ where $0<t_{0}<1$, we have that $\alpha_{i} \cap F \times\left[t_{0}, 1\right]=$ $\beta_{i} \cap F \times\left[t_{0}, 1\right]=\left\{x_{i}\right\} \times\left[t_{0}, 1\right]$ for $i=1, \cdots, n$. Then there is an isotopy of $M$ constant on $\operatorname{bd}(M)$ carrying $\alpha_{i} \cap F \times\left[0, t_{0}\right]$ to $\beta_{i} \cap F \times\left[0, t_{0}\right]$ and carrying $\alpha_{i} \cap F \times\left[t_{0}, 1\right]$ to a monotonic arc for $i=1, \cdots, n$.

Proof. By assumption there is a homeomorphism $h: F \times I \rightarrow F \times I$ carrying $\left\{\alpha_{i}: i=1, \cdots, n\right\}$ to $\left\{\beta_{i}: i=1, \cdots, n\right\}$ which leaves bd $(F) \times I \cup$ $F \times\{0\}$ fixed. By Theorem 2.1 there is an isotopy of $h$ to the identity which is constant on $\operatorname{bd}(F) \times I \cup F \times\{0\}$. Define $k: F \rightarrow F$ by $k(v)=p h(v, 1)$ for $v$ in $F$. Then it can be seen that there is an isotopy $K: F \times I \rightarrow F$ which is constant on $\operatorname{bd}(F)$ such that $k_{0}$ is the identity, $k_{1}=k^{-1}$, and $K$ is induced by our isotopy of $h$. Furthermore if we define $h_{1}: F \times I \rightarrow F \times I$ by

(1) $h_{1}(v, t)=\left(K\left(v,\left(t-t_{0}\right) /\left(1-t_{0}\right)\right), t\right)$ for $v$ in $F$ and $t$ in $\left[t_{0}, 1\right]$,

(2) $h_{1}(v, t)=(v, t)$ for $v$ in $F$ and $t$ in $\left[0, t_{0}\right]$,

it is easily seen that the homeomorphism $h_{1} h \mid \operatorname{bd}(F \times I)$ is the identity and $h_{1} h$ is isotopic to the identity under a deformation constant on bd $(F \times I)$ and level preserving on $F \times\left[t_{0}, 1\right]$. Since $h_{1} h\left(\alpha_{i} \cap\left[0, t_{0}\right]\right)=\beta_{i} \cap\left[0, t_{0}\right]$ and $h_{1} h\left(\alpha_{i} \cap\left[t_{0}, 1\right]\right)$ is monotonic for $i=1, \cdots, n$, this completes the proof of Theorem 3.1.

THEOREM 3.2. Let $\alpha$ and $\beta$ be arcs normal in $F \times I$ with $\alpha \cup \beta$ a loop. Then $\alpha$ and $\beta$ are isotopically equivalent if and only if they are homeomorphically equivalent and the loop $\alpha \cup \beta$ is nullhomotopic in $F \times I$.

Proof. We need only show that $\alpha$ and $\beta$ are isotopically equivalent if they are homeomorphically equivalent. We first find a homeomorphism $h: F \times I \rightarrow F \times I$ so that $h(\beta) \cap F \times\left[t_{1}, 1\right]=\{x\} \times\left[t_{1}, 1\right]$ for $0<t_{1}<1$ and $t_{1}$ near 1 . We may denote $h(\beta)$ by $\beta$ and $h(\alpha)$ by $\alpha$. After a deformation constant on $\operatorname{bd}(M)$, we may assume that $\alpha \cap F \times\left[t_{2}, 1\right]=\{x\} \times\left[t_{2}, 1\right]$ for $0<t_{2}<1$ and $t_{2}$ sufficiently near 1 . Let $t_{0}=$ maximum of $t_{1}$ and $t_{2}$. By Theorem 3.1 after a deformation constant on $\mathrm{bd}(M)$, we may assume that $\alpha \cap\left[0, t_{0}\right]=\beta \cap\left[0, t_{0}\right]$ and $\alpha \cap\left[t_{0}, 1\right]$ is a monotonic arc in $F \times\left[t_{0}, 1\right]$. We recall that $\beta \cap\left[t_{0}, 1\right]=\{x\} \times\left[t_{0}, 1\right]$. Now by Theorem $2.2, \alpha \cap\left[t_{0}, 1\right]$ is homeomorphically unknotted since it is a monotonic arc. Since the loop 
$\alpha \cup \beta$ is homotopic to a point and $\alpha \cap F \times\left[0, t_{0}\right]=\beta \cap F \times\left[0, t_{0}\right]$, the loop $\left(\alpha \cap F \times\left[t_{0}, 1\right]\right) \cup\left(\beta \cap F \times\left[t_{0}, 1\right]\right)$ is homotopic to a point. Since $\beta \cap F \times$ $\left[t_{0}, 1\right]=\{x\} \times\left[t_{0}, 1\right]$, the loop $p\left(\alpha \cap F \times\left[t_{0}, 1\right]\right)$ is homotopic to a point. By Theorem 2.3, there is an isotopy of $F \times\left[t_{0}, 1\right]$ constant on bd $\left(F \times\left[t_{0}, 1\right]\right)$ carrying $\alpha \cap F \times\left[t_{0}, 1\right]$ to $\beta \cap F \times\left[t_{0}, 1\right]$ and Theorem 3.2 follows.

Lemma 3.3. Let $\alpha_{i}, i=1, \cdots, n$, be a normal collection of arcs embedded in $M=F \times I$. Let $S$ be a 2-sphere embedded in $M-\bigcup_{i=1}^{n} \alpha_{i}$. Then $S$ bounds a 3-ball embedded in $M-\bigcup_{i=1}^{n} \alpha_{i}$.

Proof. Since $M$ is irreducible, $S$ bounds a 3-ball embedded in $M$. Since $\bigcup_{i=1}^{n} \alpha_{i}$ does not meet $S, \bigcup_{i=1}^{n} \alpha_{i}$ does not meet the interior of the ball bounded by $S$. Lemma 3.3 follows.

Lemma 3.4. Let $\alpha_{i}, i=1, \cdots, n$, be a normal collection of arcs embedded in $M=F \times I$. Let $A_{1}$ and $A_{2}$ be annuli properly embedded in $M-$ $\bigcup_{i=1}^{n} \alpha_{i}$ such that $A_{1}$ and $A_{2}$ each meet both $F \times\{0\}$ and $F \times\{1\}$ in a nonnullhomotopic orientable, simple loop and $A_{1} \cap F \times\{0\}=A_{2} \cap F \times\{0\}$. Then there is an isotopy of $M$ carrying $A_{1}$ to $A_{2}$ which is constant on $F \times\{0\} \cup$ $\operatorname{bd}(F) \times I \cup \bigcup_{i=1}^{n} \alpha_{i}$.

The proof of Lemma 3.4 is exactly what one would expect. One pushes $A_{1}$ off of $A_{2}$ except for $A_{1} \cap A_{2} \cap F \times\{0\}$. Then one pushes $A_{1}$ to $A_{2}$. It is impossible to follow Waldhausen's proof of 3.4 in [6] since the arcs $\alpha_{i}$ may get in the way.

ProOF. It is a consequence of Theorem 5.1 in [1] that we may assume that $A_{2}=\lambda \times I$ where $\lambda$ is a simple non-nullhomotopic loop in $F$. After a deformation, we may assume that $A_{1}$ meets $\lambda \times I$ in a collection of disjoint simple loops embedded in $\lambda \times I$ together with a number of simple arcs with their boundary contained in $\lambda \times\{0,1\}$.

If there is a simple nullhomotopic loop in $A_{1} \cap \lambda \times I$, it bounds a disk both on $\lambda \times I$ and on $A_{1}$. Thus we could find an "innermost" disk on $A_{1}$ bounded by a loop $l$ in $A_{1} \cap \lambda \times I$. Then $l$ would also bound a disk on $\lambda \times I$ and the union of these two disks would be a sphere $S$ embedded in $M-\bigcup_{i=1}^{n} \alpha_{i}$. By Lemma 3.3, $S$ bounds a ball embedded in $M-\bigcup_{i=1}^{n} \alpha_{i}$. Thus by the usual argument after a deformation constant on $\operatorname{bd}(M) \cup$ $\bigcup_{i=1}^{n} \alpha_{i}$, we may assume that $A_{1} \cap \lambda \times I$ contains no simple nullhomotopic loops and thus no arcs with both endpoints on $\lambda \times\{0\}$.

If $A_{1} \cap \lambda \times I$ contains a simple arc $\beta$ with both endpoints on $\lambda \times\{1\}, \beta$ together with a segment of $A_{1} \cap F \times\{1\}$ bounds a disk $\mathscr{D}_{1}$ on $A_{1}$. We may assume that $\mathscr{D}_{1} \cap \lambda \times I=\beta$. Now $\beta$ together with a segment of $\lambda \times\{1\}$ bounds a disk $\mathscr{D}_{2}$ on $\lambda \times I$. Now $\mathscr{D}_{1} \cup \mathscr{D}_{2}$ is a disk properly embedded in $M$. Since $\operatorname{bd}\left(\mathscr{D}_{1} \cup \mathscr{D}_{2}\right)$ lies on $F \times\{1\}$ and $F \times\{1\}$ is incompressible in $M, \operatorname{bd}\left(\mathscr{D}_{1} \cup \mathscr{D}_{2}\right)$ bounds a disk $\mathscr{D}$ embedded in $F \times\{1\}$. Thus $\mathscr{D} \cup \mathscr{D}_{1} \cup \mathscr{D}_{2}$ is a 2-sphere 
embedded in $M$. By the usual argument after a deformation constant on $\bigcup_{i=1}^{n} \alpha_{i} \cup$ bd $(F) \times I \cup F \times\{0\}$, we may assume that $A_{1} \cap \lambda \times I$ contains no simple arcs running from $\lambda \times\{1\}$ to itself. Thus $A_{1} \cap \lambda \times I$ contains at most a single arc from $\lambda \times\{0\}$ to $\lambda \times\{1\}$. Since $\lambda$ is two-sided in $F, A_{i} \cap \lambda \times I$ consists of a collection of simple non-nullhomotopic loops.

Let $l$ be the loop on $A_{1}$ which bounds a subannulus $A$ of $A_{1}$ which meets $\lambda \times I$ in $l$. Let $M^{*}=F_{1} \times I$ be the manifold obtained by splitting $M$ along $\lambda \times I$ and $P: M^{*} \rightarrow M$ the natural projection. Let $A^{*}$ be the annular component of $P^{-1}(A)$. Since $H_{2}\left(F_{1} \times I, F_{1} \times\{1\} \cup \operatorname{bd}\left(F_{1} \times I\right) ; Z_{2}\right)=0, A^{*}$ bounds a finite 3-chain in $C_{3}\left(F_{1} \times I, F_{1} \times\{1\} \cup\right.$ bd $\left.F_{1} \times I ; Z_{2}\right)$. Denote the 3-manifold representing one such chain by $X_{1}$ and $\operatorname{cl}\left(M^{*}-X_{1}\right)$ by $X_{2}$. We assume $X_{2}$ contains $F_{1} \times\{0\}$. Then $X_{1} \cap X_{2}=A^{*}$. Thus Van Kampen's theorem promises the diagram of groups shown in Figure 1 where all maps shown are induced by inclusion.

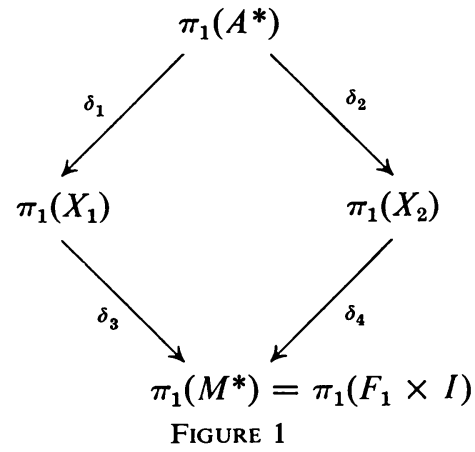

Note that $\delta_{1}$ and $\delta_{2}$ are monomorphisms, and $\delta_{4}$ is onto since $X_{2}$ contains $F_{1} \times\{0\}$. It follows from 4.2 in [2] that $\delta_{1}$ is onto. Thus by 3.1 in [1] there is a homeomorphism $h: X_{1} \rightarrow A^{*} \times I$ carrying $A^{*}$ to $A^{*} \times\{0\}$. Thus it is not difficult to push $A_{1}$ across $P\left(X_{1}\right)$ to reduce the number of loops in $A_{1} \cap \lambda \times I$ since $P\left(X_{1}\right)$ does not meet $\bigcup_{i=1}^{n} \alpha_{i}$. If there is only one such loop, we could push $A_{1}$ across $P\left(X_{1}\right)$ onto $\lambda \times I$. This completes the proof of Lemma 3.4.

THEOREM 3.5. Let $F$ be a surface other than the torus, projective plane or 2-sphere and $\left\{\alpha_{i}: i=1, \cdots, n\right\}$ and $\left\{\beta_{i}: i=1, \cdots, n\right\}$ normal collections of arcs embedded in $M=F \times I$ which are homeomorphically equivalent. Let $p \operatorname{bd}\left(\alpha_{i}\right)=p \operatorname{bd}\left(\beta_{i}\right)=\left\{x_{i}\right\}$ for $i=1, \cdots, n$. Let $\lambda_{1}, \cdots, \lambda_{m}$ be a collection of disjoint, non-nullhomotopic, simple, orientable loops properly embedded in $F-\left\{x_{i}: i=1, \cdots, n\right\}$ such that each component of $F-\bigcup_{j=1}^{m} \lambda_{j}$ contains at most one of the $x_{i}$. Assume that $\lambda_{j} \times\{0\}$ is freely homotopic to $\lambda_{j} \times\{1\}$ in $M-\bigcup_{i=1}^{n} \alpha_{i}$ and in $M-\bigcup_{i=1}^{n} \beta_{i}$. If the loop $\alpha_{i} \cup \beta_{i}$ is nullhomotopic for $i=1, \cdots, n$, then the collections $\left\{\alpha_{i}: i=1, \cdots, n\right\}$ and $\left\{\beta_{i}: i=1, \cdots, n\right\}$ are isotopically equivalent. 
Proof. The proof of this theorem is almost a triviality since we have Theorem 3.2 and Lemma 3.4. We simply arrange to have each pair of normal arcs lie in its own product manifold and then apply Theorem 3.2.

It is a consequence of the generalization of Dehn's lemma in [5] that $\lambda_{j} \times\{0,1\}$ bounds annuli $A_{j}$ in $F \times I-\bigcup_{i=1}^{n} \alpha_{i}$ and $B_{j}$ in $F \times I-\bigcup_{i=1}^{n} \beta_{i}$ for $j=1, \cdots, m$. Using standard techniques we may assume that the $A_{j}$ are pairwise disjoint as are the $B_{j}$. It is a consequence of Theorem 5.2 in [1] that after a homeomorphism we may assume $B_{j}=\lambda_{j} \times I$ for $j=1, \cdots, k$. It is a consequence of Theorem 2.5 that, after a deformation constant on $\operatorname{bd}(M)$, we may assume $A_{j}=\lambda_{j} \times I$ for $j=1, \cdots, m$. Let $h: M \rightarrow M$ be a homeomorphism such that $h\left(\alpha_{i}\right)=\beta_{i}$ for $i=1, \cdots, n$, and $h \mid F \times\{0\} \cup$ $\operatorname{bd}(F) \times I=$ id. If $h \mid \bigcup_{j=1}^{m} \lambda_{j} \times I=$ id, the theorem would follow from 3.2. It is a consequence of Lemma 3.4 that we may assume that $h\left(\lambda_{j} \times I\right)=$ $\lambda_{j} \times I$ after a deformation constant on $\bigcup_{j=1}^{n} \beta_{j} \cup F \times\{0\} \cup \operatorname{bd}(F) \times I$. After a deformation constant outside of a small neighborhood of $\bigcup_{j=1}^{m} \lambda_{j} \times I$, we can assume $h \mid \lambda_{j} \times I=\mathrm{id}$ for $j=1, \cdots, m$. This completes the proof of Theorem 3.5.

We will denote the Euler characteristic of a manifold $F$ by $\chi(F)$.

THEOREM 3.6. Let $\left\{\alpha_{i}: i=1, \cdots, n\right\}$ and $\left\{\beta_{i}: i=1, \cdots, n\right\}$ be normal collections of arcs, embedded in $M=F \times I$, which are homeomorphically equivalent. We suppose that $\chi(F)<0$ and that for every simple loop $\lambda$ in $F-\left\{x_{i}: i=1, \cdots, n\right\}, \lambda \times\{0\}$ is freely homotopic to $\lambda \times\{1\}$ in $M-\bigcup_{i=1}^{n} \alpha_{i}$ and in $M-\bigcup_{i=1}^{n} \beta_{i}$. Then the collections $\left\{\alpha_{i}: i=1, \cdots, n\right\}$ and $\left\{\beta_{i}: i=\right.$ $1, \cdots, n\}$ are isotopically equivalent.

Proof. Theorem 3.6 will follow from Theorem 3.5 when we show that loop $\alpha_{i} \cup \beta_{i}$ is nullhomotopic for $i=1, \cdots, n$. Actually one could show that after a homeomorphism the $\alpha_{i}$ lie in disjoint product disks for $i=$ $1, \cdots, n$, but we do not need to prove this.

There exist non-nullhomotopic simple loops $\lambda_{1}$ and $\lambda_{2}$ properly embedded in $F$ whose intersection is the single point $x_{1}$. We may suppose that if $\left[\lambda_{1}\right]^{n_{1}}=\left[\lambda_{2}\right]^{n_{2}}, n_{1}=n_{2}=0$ since $\chi(F)<0$. We let $R$ be an annulus or möbius band embedded in $F$ and containing $\lambda_{1}$ in its interior. We may suppose that $R$ can be deformation retracted to $\lambda_{1}$. Now the loops in $\operatorname{bd}(R \times\{0\})$ are freely homotopic to the corresponding loops in $\operatorname{bd}(R \times\{1\})$ in $M-\alpha_{1}$ and in $M-\beta_{1}$. We suppose $R$ is a möbius band. Then $\operatorname{bd}(R) \times\{0\}$ and $\operatorname{bd}(R) \times\{1\}$ bound an annulus $B$ in $M-\beta_{1}$ by the generalized Dehn's lemma in [5]. Theorem 5.1 in [1] implies the existence of a homeomorphism $h: F \times I \rightarrow F \times I$ such that $h(B)=\operatorname{bd}(R) \times I$. We may think of $h\left(\beta_{1}\right)$ as $\beta_{1}$, $h\left(\alpha_{1}\right)$ as $\alpha_{1}$, and $h(A)$ as $A$ and proceed assuming that $B=\operatorname{bd}(R) \times I$. Similarly $\operatorname{bd}(R)$ bounds an annulus $A$ in $M-\alpha_{1}$. By Theorem 2.5 we may assume that $A=\operatorname{bd}(R) \times I$. Thus the loop $p\left(\alpha_{1} \cup \beta_{1}\right) \subset R$ represents 
$\left[\lambda_{1}\right]^{n_{1}}$ for some integer $n_{1}$. If $R$ was an annulus, we would have reached the same conclusion by a similar argument. Similarly we can conclude that the loop $p\left(\alpha_{1} \cup \beta_{1}\right)$ represents an element $\left[\lambda_{2}\right]^{n_{2}}$ in $\pi_{1}(F)$. It follows that the loop $\alpha_{1} \cup \beta_{1}$ is nullhomotopic in $M$. Similarly the loop $\alpha_{i} \cup \beta_{i}$ is nullhomotopic in $M$ for $i=1, \cdots, n$. Thus Theorem 3.6 follows.

One cannot help but notice that our conditions for isotopic equivalence of collections of knotted arcs enable us to confine pairs of arcs to product manifolds. It would be interesting to find conditions implying isotopic equivalence of normal families of arcs which did not allow us to do this. One might also hope to obtain results in manifolds other than $F \times I$ and $S^{\mathbf{3}}$.

\section{REFERENCES}

1. E. M. Brown, Unknotting in $M^{2} \times 1$, Trans. Amer. Math. Soc. 123 (1966), 480-505. MR 33 \#6640.

2. E. M. Brown and R. H. Crowell, The augmentation subgroup of a link, J. Math. Mech. 15 (1966), 1065-1074. MR 33 \#4920.

3. C. D. Feustel, Isotopic unknotting in F $\times 1$, Trans. Amer. Math. Soc. (to appear).

4. J. Martin and D. Rolfsen, Homotopic arcs are isotopic, Proc. Amer. Math. Soc. 19 (1968), 1290-1292. MR 38 \#719.

5. A. Shapiro and J. H. C. Whitehead, A proof and extension of Dehn's lemma, Bull. Amer. Math. Soc. 64 (1958), 174-178. MR 21 \#2242.

6. F. Waldhausen, On irreducible 3-manifolds which are sufficiently large, Ann. of Math. (2) 87 (1968), 56-88. MR 36 \#7146.

Department of Mathematics, Virginia Polytechnic Institute and State UNIVERSITY, BLACKSBURG, VIRGINIA 24061 